\title{
Design of an Ergonomic Gaming Console
}

\author{
Sachin V. Bhat ${ }^{1, a}$, Nikhil Kajaria ${ }^{2, b}$, Anant Kanoria ${ }^{3, c}$, \\ Hridhay Rajkumar $^{4, \mathrm{~d}}$, Sunil R Yalamalle ${ }^{5, \mathrm{e}}$ \\ ${ }^{12345}$ Industrial Engineering and Management, R.V. College of Engineering, Bengaluru - 560059. \\ asachinvasanthbhat@gmail.com, bnikhil.11592@gmail.com, 'canantkanoria@gmail.com, \\ dhridhayrajkumar@gmail.com, ${ }^{\mathrm{e}}$ sunilry@rvce.edu.in
}

Key words-Anthropometry, Design, Ergonomics, Keyboard

\begin{abstract}
The average gamer faces a number of musculo-skeletal injuries due to bad design of keyboards, consoles, mice, workspace layout and a number of other factors. This study aims at minimizing injuries and maximizing human comfort by using Ergonomic principles in the design of the console, which also happens to be the main interactive device between the gamer and the computer. Hence the objective of this study would be to design a keyboard to optimize the comforts of using a gaming console for computer games played on the keyboard, through the sciences of anthropometry and ergonomics.
\end{abstract}

\section{Introduction}

The average gamer faces a number of musculo-skeletal injuries due to bad design of keboards, consoles, mice, workspace layout and a number of other factors. This study aims at minimizing injuries and maximizing human comfort by using Ergonomic principles in the design of the keyboard, which also happens to be the main interactive device between the gamer and the computer. Hence the objective of this study would be to design a keyboard to optimize the comforts of using the gaming console for computer games played on a keyboard, through the sciences of anthropometry and ergonomics.

\section{Literature Review}

The objective of optimization in a traditional keyboard is to arrange the characters on the keys in a manner that is most efficient in terms of typing time and effort [1]. It has been observed that the utilization of this traditionally designed keyboard for prolonged gaming purposes leads to musculoskeletal disorders arising from repetitive stress injuries. Research has been done extensively on repetitive stress injuries associated with extensive use of the keyboard. Repetitive strain injuries are reaching epidemic levels among workers who perform heavy schedules of rapid alternating movements or repetitive, sustained, coordinated movements [2].Work related Musculoskeletal disorders of the hand and wrist are associated with the longest absences from work and leads to loss of productivity [3].

The unfortunate effects of gaming can, however, be more direct: in 1991, a type of pressure injury seen in adolescent gamers was described and christened 'Nintendonitis'. After an enthusiastic and prolonged session with a new games console (in this case, a Nintendo 64), the patient developed a punched-out ulcer on her right palm [4]. More than 13 years later, a very similar phenomenon was described in The Lancet, this time dubbed 'PlayStation 2 (PS2) Thumb' [5]; PS2 thumb was later described in 'epidemic' proportions in The South African Medical Journal [6] Confusingly, the term 'Nintendinitis' (compared to 'Nintendonitis') was also coined in 1990 to describe a repetitive straintype injury affecting the wrist and elbow [7]. Upon the advent of the Wii, however, PlayStation thumb and Nintendintis were eclipsed by altogether different problems. 
Due to these widespread injuries, the focus has been shifting, in recent times, to more scientific design of keyboards and consoles using ergonomic principles of design. With gaming injuries forming a large proportion of all keyboard-related injuries, this study focuses on designing an ergonomic gaming keyboard.

Anthropometric data forms the basis of this ergonomic intervention. The dimensions for designing products are obtained from anthropometric data. Few studies pertaining to the collection of anthropometric data of Indians exist and these cannot be used for designing a keyboard for gaming purposes. This study also aims to establish a hand anthropometric database.

To achieve better efficiency of performance, more human comfort and to reduce musculoskeletal injury, it is necessary to design the hand tools and equipment keeping in consideration the operator's capabilities and limitations. The design of equipment is always a compromise between the operator's biological needs, which are determined by the ergonomics guidelines, and physical requirements of the equipment [8] [9]. In this regard, the basic information required is the anthropometric body dimensions of the users of tools and equipment.

\section{Diagnosis of Problem}

Before proceeding further on the design, it is imperative to analyze the various negative repercussion of using the existing keyboard and game consoles for gaming which are listed as below.

- Work related Musculoskeletal disorders(WMSD)- WMSD of the hand and wrist are associated with the longest absences from work and leads to loss of productivity [3].

- Nintendonitis-Thumb swelling occurs due to Repetitive Stress Injury(RSI) to the thumb from the continuous and excessive use of a game controller [4].

- PlayStation Thumb-PlayStation thumb is a RSI resulting from continuous playing of PlayStation games for many hours and can manifest as pain in the thumbs and blisters on the tips of the thumbs. Other associated symptoms include pain, stiffness, swelling, numbness and tingling of the hands, wrists, elbows, shoulders, back or neck. [6].

- Carpal Tunnel Syndrome - It is a condition in which there is pressure on the median nerve -the nerve in the wrist that supplies feeling and movement to parts of the hand. It can lead to numbness, tingling, weakness, or muscle damage in the hand and fingers. Using the computer Keyboard is most common cause of carpal tunnel [10].

\section{Methods}

To realize the objective of optimization of user comforts in the gaming console, it is necessary to do a systematic study of ergonomic and anthropometric data pertaining to a wide cross section of end users of this gaming software.

Next, the different keys involved in the gameplay are identified from the 'classic' keyboard controls. The keys which are used in game play are segregated based on the finger employed to operate the key during game play and a separate colour is assigned to each key for easy identification of key during gameplay as shown in Table I and Table II.

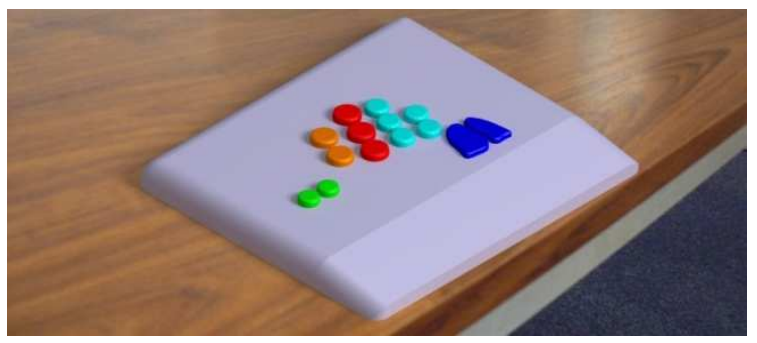

Figure 1 (a): Left Hand Console.

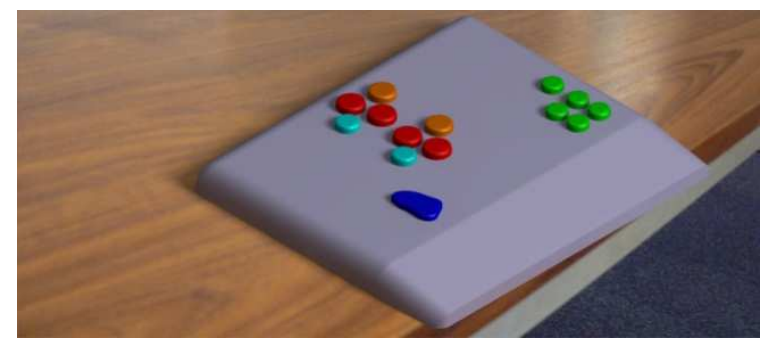

Figure 1 (b): Right Hand Console 


\section{Table I}

Colour Code Explanation For Left Hand Console.

\begin{tabular}{|l|l|l|l|}
\hline Serial No. & Colour Code & Finger Employed & Number of Keys Operable \\
\hline 1. & Green & Little Finger & 2 \\
\hline 2. & Orange & Ring Finger & 2 \\
\hline 3. & Red & Middle Finger & 3 \\
\hline 4. & Turquoise & Index Finger & 2 \\
\hline 5. & Blue & Thumb & 1 \\
\hline
\end{tabular}

Table II

Colour code explanation For Right hand console.

\begin{tabular}{|l|l|l|l|}
\hline Serial No. & Colour Code & Finger Employed & Number of Keys Operable \\
\hline 1. & Green & Little Finger & 5 \\
\hline 2. & Orange & Ring Finger & 2 \\
\hline 3. & Red & Middle Finger & 4 \\
\hline 4. & Turquoise & Index Finger & 5 \\
\hline 5. & Blue & Thumb & 2 \\
\hline
\end{tabular}

The locations and sizes of the keys are fixed on the keyboard such that they provide the most sensible option during gameplay. These tables of keys is an exhaustive list and thus, no further keys will be included as they are meant for the purpose of playing the game smoothly only. This will act as a secondary peripheral to the primary keyboard already being used to control the desktop, or installed within the laptop.

\section{Data Collection}

The sample population consists of individuals from different parts of India, aged between the years of 17 and 24. Data collection is carried out in the following steps. Firstly, the candidate is asked to place his/her hand on a flatbed scanner and 3 images of the candidate are taken, in the positions of condensed hand, neutral span hand and crouched hand.

The name and sex of the candidate are collected spontaneously and the images are stored after verifying the feasibility and usability of the images. In case the images are not feasible, the subject is asked to get his/her hand scanned again.

Then the images are rendered in AutoCAD and measurements are taken accurately for the different anthropometric parameters and tabulated for both men and women. These tabulated anthropometric parameters are then used to design the location of various keys on the game console along with their various degrees of motion.
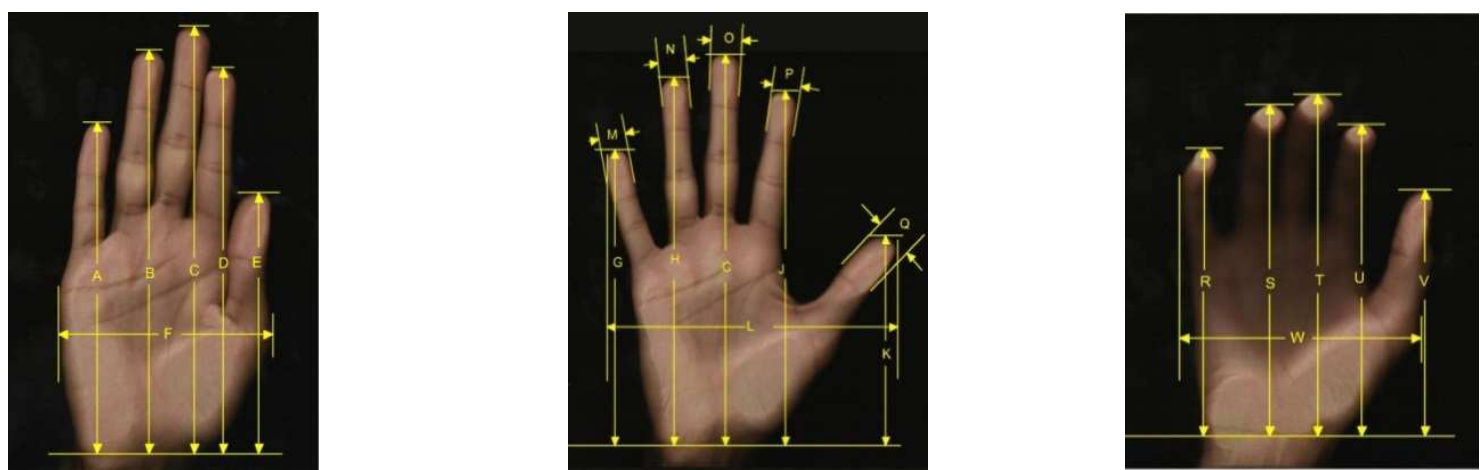

Fig 2(a) Condensed Position. Fig 2(b) Neutral Span Position. Fig 2(c) Neutral Crouch Position. 


\section{Parameters}

The various parameters that were quantified from the sample population were as follows:

- Finger end thickness: In Fig. 2(b), the hand is kept flat against the flatbed scanner and spanned to the relaxation position. The distance between one end of the tip section of each finger to the other end is taken as the measure for this.

M: Small finger distal phalanx width. N: Ring finger distal phalanx width. O: Middle finger distal phalanx width. P: Index finger distal phalanx width. Q: Thumb finger distal phalanx width.

- Hand length (Condensed): In Fig. 2(a), the hand is kept flat against the flatbed scanner in the condensed position with the thumb-tip pointing upward and the body of the thumb touching the palm. The perpendicular distances measured from the wrist crease directly below the pad of muscle at the base of the thumb to the tip of the distal phalanx of each finger are the different lengths of each finger in condensed position.

A: Perpendicular distance from tip of distal phalanx of little finger to wrist crease. B: Perpendicular distance from tip of distal phalanx of ring finger to wrist crease. C: Perpendicular distance from tip of distal phalanx of middle finger to wrist crease. D: Perpendicular distance from tip of distal phalanx of index finger to wrist crease. E: Perpendicular distance from tip of distal phalanx of thumb to wrist crease.

- Hand length (Neutral span): In Fig 2(b), the hand is kept flat against the flatbed scanner with the fingers spread outward in a neutral position. The perpendicular distances measured from the wrist crease directly below the pad of muscle at the base of the thumb to the tip of the distal phalanx of each finger are the different lengths of each finger in the neutral span position.

G: Perpendicular distance from tip of distal phalanx of little finger to wrist crease. H: Perpendicular distance from tip of distal phalanx of ring finger to wrist crease. C: Perpendicular distance from tip of distal phalanx of middle finger to wrist crease. J: Perpendicular distance from tip of distal phalanx of index finger to wrist crease. K: Perpendicular distance from tip of distal phalanx of thumb to wrist crease.

- Hand length (Neutral crouch): In Fig. 2(c), the hand is kept flat against the flatbed scanner with the fingers spread outward in a neutral position and the palm arched outward to create a slight " $C$ " with the hand. The perpendicular distances measured from the wrist crease directly below the pad of muscle at the base of the thumb to the tip of the distal phalanx of each finger are the different lengths of each finger in the neutral crouch position. R: Perpendicular distance from tip of distal phalanx of little finger to wrist crease. S: Perpendicular distance from tip of distal phalanx of ring finger to wrist crease. T: Perpendicular distance from tip of distal phalanx of middle finger to wrist crease. U: Perpendicular distance from tip of distal phalanx of index finger to wrist crease. V: Perpendicular distance from tip of distal phalanx of thumb to wrist crease.

- Hand breadth across thumb (Condensed): The hand is kept flat against the flatbed scanner in the condensed position with the thumb-tip pointing outward and the body of the thumb touching the palm. The distance measured from the end of the palm to the outward of the thumb is the breadth of hand across thumb for condensed position.

F: Horizontal distance measured between the tip of $1^{\text {st }}$ metacarpal and the tip of $5^{\text {th }}$ metacarpal.

- Hand breadth across thumb (Neutral span): The hand is kept flat against the flatbed scanner with the fingers spread outward in a neutral position. The distance measured from the outward edge of the little finger to the opposite outward end of the thumb is the breadth of the hand across thumb for neutral span position. L: Horizontal distance measured between tip of distal phalanx of little finger and the distal phalanx of the thumb.

- Hand breadth across thumb (Neutral crouch):The hand is kept flat against the flatbed scanner with the fingers spread outward in a neutral position and the palm arched outward to create a slight " $C$ " with the hand. The distance measured from the outward edge of the little finger to the opposite outward end of the thumb is the breadth of the hand at metacarpal for neutral crouch position. 
W: Horizontal distance measured between tip of distal phalanx of little finger and the distal phalanx of the thumb.

\section{Design}

The materials preferred for this design are regular polymers. The main idea of this project was to create a split game console to provide flexibility for the gamer to incline the game console to a position comfortable to him depending on the height of the work surface. The dynamic data of the hand in a crouched position was taken as a reference. The image of the hand in the crouched position is raster in AutoCAD and an outline of the various keys and the key shapes are drawn from the anthropometric statistical model drawn. All the edges of the body panel are filleted to avoid sharp edges which cause discomfort during repetitive usage. A small incline of about 15 degrees is provided at palm rest for better comfort. The diameters of the keys were inferred from the anthropometric data. The shape of the key that is operated by the thumb is customized to suit the profile of the thumb. Each key is color coded. This helps in easy identification of the keys during game play. The keys are filleted all around to avoid sharp edges coming in contact with the finger. A special contour is provided on the top surface of the key as to accommodate the shape of the finger.

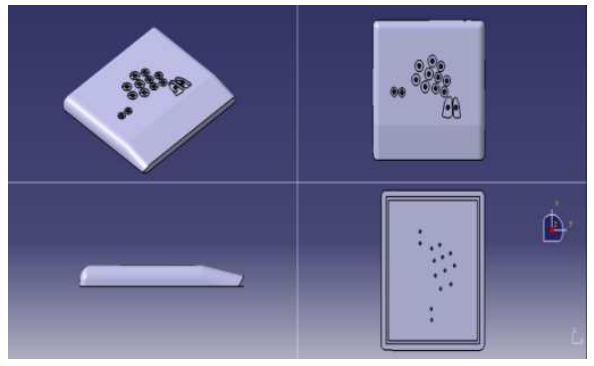

Fig 3(a): Model of the body panel

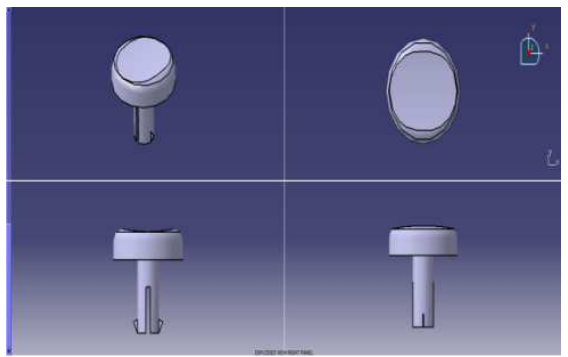

Fig 3(b): Profile of the Key

\section{Conclusion}

The data thus analyzed will be used for designing the gaming console so as to give optimum comforts to the user. The data being collected through extensive field work which would take over 2 months to complete. To eliminate repetitive stress injury, the keyboard will be padded by a flexible polystyrene hand rest around the keyboard with the right contours in keeping with the ergonomic parameters evolved from the analysis of the data. The force that is applied to the keys to activate it is negligible and hence the keys can be assumed to be feather touch.

Further to enhance the user friendliness of the keyboard, attractive colour coding is adopted in the system. This will facilitate the user to easily perceive the various keys in the gaming console thus increasing user friendliness.

\section{References}

[1] Priyendra S. Deshwal et al, "Ergonomic Design of Optimal Hindi Keyboard for Convenient Use".

[2] Byl N, Wilson F, Merzenich M, Melnick M, Scott P, Oakes A, McKenzie A.J Orthop Sports Phys Ther. 1996 Apr; 23(4):234-44.

[3] Ann E. Barr, PT, PhD, Mary F. Barbe, PhD, and Brian D. Clark, PhD, "Work-related musculoskeletal disorders of the hand and wrist: epidemiology, pathophysiology, and sensorimotor changes.'J Orthop Sports Phys Ther. 2004 October; 34(10): 610-627.

[4] Siegel,"Nintendonitis",IMOrthopedics. 1991 Jul; 14(7):745.

[5] Vaidya HJ, "Playstation thumb."Lancet. 2004 Mar 27; 363(9414):1080.

[6] Karim SA, "Playstation thumb--a new epidemic in children."S Afr Med J. 2005 Jun; 95(6):412. 
[7] Brasington R, “Nintendinitis.”N Engl J Med. 1990 May 17; 322(20):1473-4.

[8] Das, B., Grady, R.M., 1983. "Industrial workplace layout design: an application ofengineering anthropometry.” Ergonomics 26 (5), 433e447.

[9] Das, B., Sengupta, A.K., 1996. "Industrial workstation design: a systematic ergonomic sapproach.” Applied Ergonomics 27 (3), 157e163. 